\section{ECA Rule Condition}

Mikael Berndtsson and Jonas Mellin University of Skövde, Skövde, Sweden

\section{Definition}

An ECA rule condition is either a database query, a logical expression, or a call to a subprogram (function or method) executing arbitrary code returning true or false. If database queries are employed, then a nonempty set is equivalent to true, and an empty set is equivalent to false.

\section{Key Points}

A key issue of an ECA rule condition is that it ought to take parameters carried by the event triggering the rule that,in turn, evaluates the con- dition. The condition either returns a set (as a result of an SQL query) or a Boolean value (as a result of evaluation of the logical expression or the execution of the subprogram). An empty set is equal to false, and a nonempty set is equal to true. Another key issue is that results of the condition evaluation can be used to optimize rule action execution in many cases. Thus, there is a need to pass parameters from the condition evaluation to the rule action execution.

\section{Cross-References}

- ECA Rule Action

- ECA Rules 